\title{
Multi-PoP Network Slice Deployment: A Feasibility Study
}

\author{
Polychronis Valsamas*, Panagiotis Papadimitriou*, Ilias Sakellariou*, Sophia Petridou*, Lefteris Mamatas*, \\ Stuart Clayman ${ }^{\dagger}$, Francesco Tusa ${ }^{\dagger}$ and Alex Galis ${ }^{\dagger}$ \\ * Department of Applied Informatics \\ University of Macedonia \\ Thessaloniki, Greece \\ \{xvalsama, papadimitriou, iliass, spetrido, emamatas\}@uom.edu.gr \\ ${ }^{\dagger}$ Department of Electronic and Electrical Engineering \\ University College London \\ London, UK \\ \{s.clayman, francesco.tusa, a.galis\}@ucl.ac.uk
}

\begin{abstract}
Network slicing is seen as a key enabler for meeting the diverse network service requirements, which stem from the transition to 5G. Furthermore, network slicing provides inherent support for multi-tenancy, enabling network providers to slice their infrastructure and resell it to a large number of tenants. Most existing work on slicing has been focused on certain mechanisms (e.g., slice embedding) and architecture specifications. As such, the performance and scalability with network slice instantiation has not been studied in depth. These aspects are even more critical in the case of slice deployments across multiple Points-of-Presence (PoP), since the various slice components should be stitched together for the end-to-end slice instantiation.

In this paper, we present the design and prototype implementation of a network slicing architecture, based on which we perform a feasibility study of network slicing using multiple experimental infrastructures. Our prototype implementation supports all the required functionality for slice instantiation, such as resource discovery, slice embedding, resource provisioning, link setup, and inter-PoP slice segment stitching. Our experimental results corroborate the feasibility of multi-PoP network slicing. We further gain useful insights on slice instantiation performance and scalability.
\end{abstract}

\section{INTRODUCTION}

Network slicing has been recently promoted as a key enabler for leasing service-tailored bundles of computer, network, and storage resources, which are often termed as verticals [1], [2]. This essentially fosters the integration of existing and novel network services into the infrastructure, as well as the co-existence of multiple services with significantly different requirements in terms of bandwidth, delay, resilience and/or security. Network Function Virtualization (NFV) [3], [4] and Software-Defined Networking (SDN) [5], [6] comprise some of the main enablers for network slicing.

Network slicing is usually not limited to the provisioning of isolated resource bundles, but it also encompasses resource management and orchestration primitives on per-slice-basis. As such, tenants are enabled to exercise fine-grained control and management on their leased slices, with minimal provider interventions. This level of control can be attained, e.g., by deploying a dedicated Virtualized Infrastructure Manager (VIM) per slice, as advocated by the NECOS project [2], [7]. Alternatively, independent slice control could be enabled through a shim layer on top of a shared VIM. Since the latter introduces high complexity, we assume a VIM-on-demand slicing model in the rest of this paper [8].

In practice, the concurrent deployment and operation of network slices on top of shared infrastructures poses the need for diverse functionality spread across different layers of the network slicing architecture. For example, network slicing requires mechanisms to advertise, discover, select and allocate resources for slice creation. In the case of slices spanning multiple Points-of-Presence (PoP) or different administrative domains, additional mechanisms are required for stitching together the different slice segments in order to instantiate the slice. While the efficiency of certain slicing mechanisms, e.g., slice embedding [9], is well understood, the feasibility of a multi-PoP slice deployment has not been studied so far, to the best of our knowledge. Existing prototype implementations mainly pertain to network (function) virtualization [10], [11], and are typically limited to single-PoP deployments.

Along these lines, we conduct a feasibility study of multiPoP network slice deployment to assess the timescales at which slices can be provisioned and further identify potential scalability bottlenecks. This study is carried out on top of geographically dispersed experimental infrastructures, which essentially offer a realistic setup for the slice provisioning performance evaluation. Our feasibility study is carried out using a prototype implementation of a network slicing architecture, which addresses the main needs of the network slice deployment, i.e., resource discovery, slice embedding, resource provisioning, tunnel setup, and inter-PoP slice segment stitching. Additional support for service deployment and resource monitoring provides the necessary means for quantifying the network slicing gains on certain applications or services.

In the following, Section II provides an overview of the NECOS network slicing architecture, on which our feasibility study relies upon. Section III discusses a proof-of-concept implementation for network slice instantiation with detailed descriptions of the individual steps taken for multi-PoP slice deployment. In Section IV, we present our experimental results, and finally in Section V, we highlight our conclusions and give future work directions. 


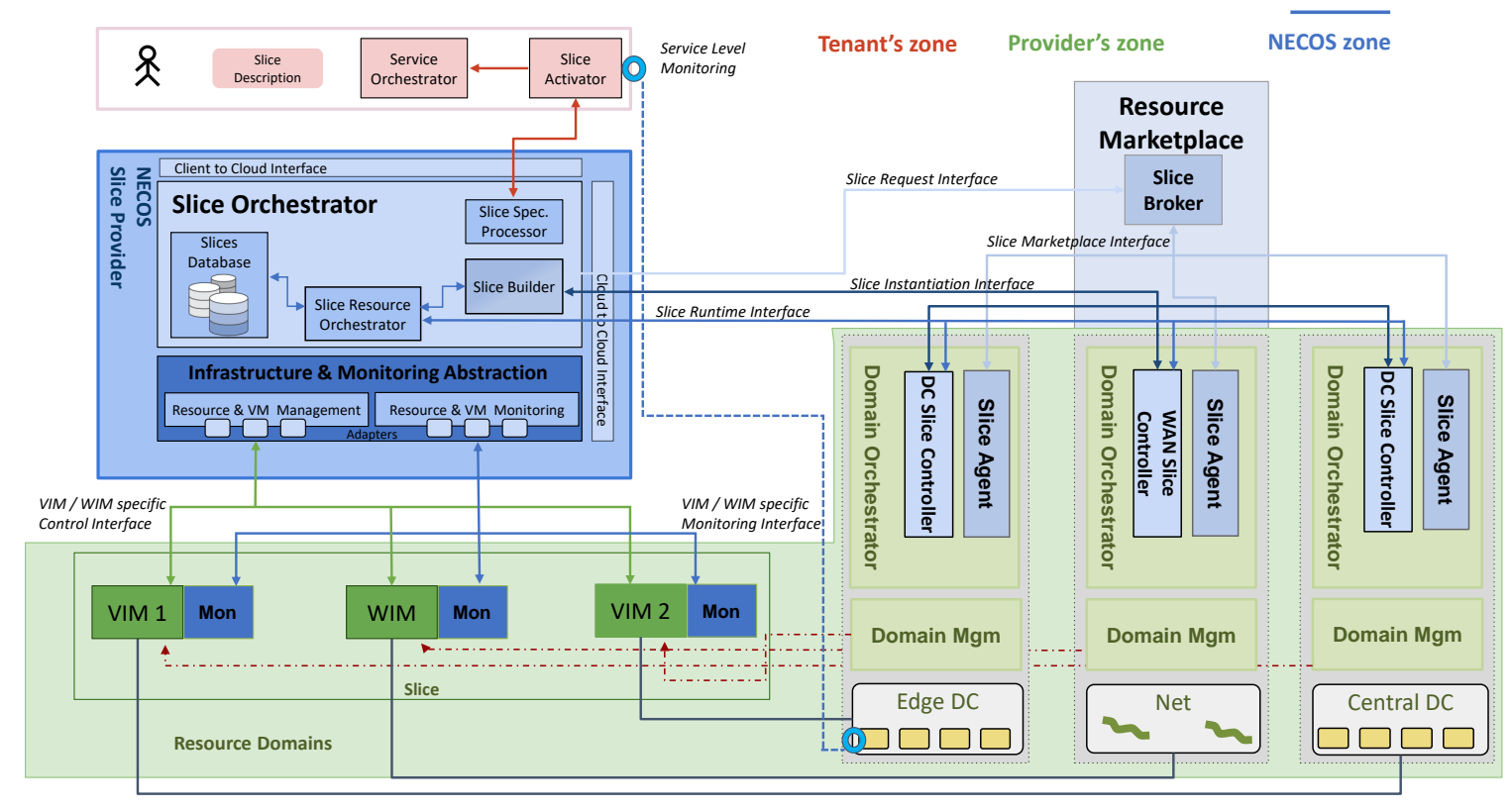

Fig. 1: NECOS architecture

\section{Network Slicing Architecture}

The proposed feasibility study relies on a prototype implementation of the network slicing architecture introduced by the NECOS project [2]. The NECOS architecture aims at creating slices by dynamically discovering and allocating resources, that span over multiple PoP or administrative domains. To achieve this, it primarily considers the following three roles:

- the NECOS Slice Provider, which is part of the NECOS ecosystem and is responsible to deploy and operate slices, based on the Tenant's request;

- the Slice Broker, which is responsible for dynamic resource discovery and acts at the core of the Marketplace;

- the DC and WAN Providers that offer either Data Center (DC), or network (WAN) resources, which will become the slice-segments of the deployed end-to-end slices.

Obviously, an administrative authority can assume more than one roles, e.g., an operator can offer a number of both DC and WAN resources and act as the NECOS Slice Provider at the same time.

Besides the roles, the NECOS architecture consists of a number of functional components that inter-operate to deliver the end-to-end slice requested by the tenant [7], as depicted in Fig. 1. Such a distributed system approach aims at offering flexibility in adapting to the needs of the different participants in the NECOS ecosystem and "separating concerns" during the implementation.

A tenant triggers a slice request in the Slice Activator, which is, in essence, a Graphical User Interface (GUI) designed to receive the slice specifications. These could be deducted by the service graph, i.e., a description of the service hosted by the slice, along with any geographic (e.g., edge cloud location to host a service function) and resource specific constraints.
This partial description of the slice is further refined by the Slice Specification Processor, that will introduce in the slice specification the slice graph, i.e., slice-segments and their necessary connections, along with a mapping between the latter and the service components.

The Slice Builder receives this specification and is responsible for: (i) initiating the resource discovery process in the Marketplace, (ii) deciding the final resource selection based on the available slice-segment received from the former, (iii) resource instantiation through contacting the $D C$ Controllers of each slice-segment, and finally, (iv) communicating this information to the Slice Resource Orchestrator in order to finalise the end-to-end slice creation and to proceed with the related activation.

The Slice Broker is the main Marketplace component. Its role consists in receiving a slice request in the form of a "partial" slice description, decomposing it into a number of single slice-segment resource requests addressed to the appropriate $D C$ and WAN Slice Agents, and compiling a response back to the Builder. This response includes alternative proposals for each slice-segment from providers that hold the resources to accommodate them. Each proposal can potentially include information regarding cost or quality characteristics (among others) that will be used by the Builder to make the overall decision on the "best" end-to-end slice configuration.

Two highly interconnected components reside on the resource providers' domain. The Slice Agent, responsible for replying to Broker requests by checking current resource availability, and the DC or WAN Controller. A controller is responsible for creating a slice-segment, i.e., for managing the provider's resources that will be offered to the NECOS ecosystem; allocating resources on-demand as requested by the 


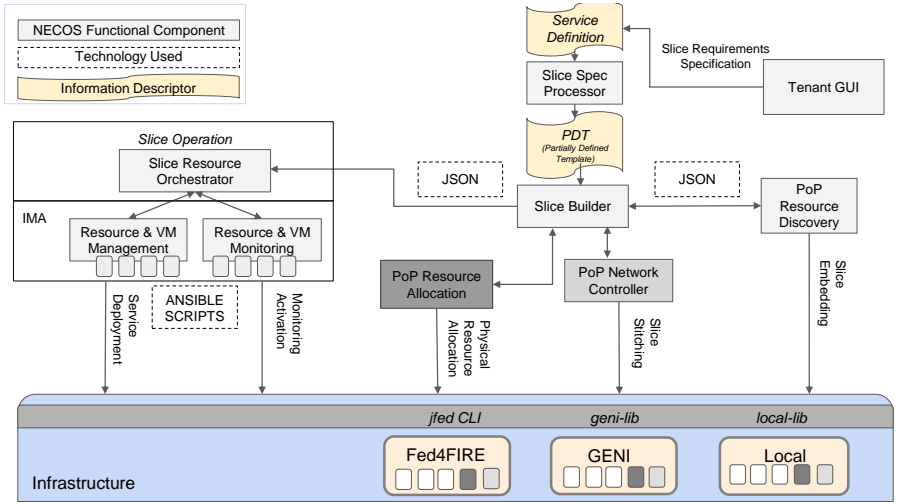

Fig. 2: Slice workflow overview

Builder; deploying on-demand VIMs and monitoring services for the slice-segment; and for any end-of-life operations, e.g., slice-segment decommission.

The Slice Resource Orchestrator (SRO) manages the lifecycle of all the (stitched) slice-segments and orchestrates the service elements across the end-to-end slice, i.e., it performs the placement and embedding of VMs into the resource domains. These actions are performed according to the monitoring information provided by the Infrastructure and Monitoring Abstraction (IMA) component. The IMA is a uniform abstraction layer introduced in order to hide the specific technological implementation of the VIM / WAN Infrastructure Manager (WIM) and monitoring subsystems in each slice-segment. A uniform northbound interface offered by IMA, allows the SRO to perform his specific functions in an abstracted way: a technology specific, pluggable adaptation layer to different VIMs/ WIMs and monitoring systems is in place at the southbound of the IMA to implement the required abstraction.

Finally, the Slice Database is the main component for storing the information related to the different end-to-end slices.

\section{PRototype ImPlementation}

In this section, we present our prototype realizing slice deployment in consistency to the NECOS approach. In comparison to the NECOS architecture, we focus on a multi-PoP rather than on a multi-provider environment. As we show in Fig. 2, our implementation supports a simplistic Marketplace operation: the Slice Broker acts as a Broker Agent as well, being responsible to report on the resource availability in the different PoPs. Along these lines, we replaced the above two entities with a new component called PoP Resource Discovery. The slice embedding operation over multiple Infrastructure and WAN providers is complex enough to deserve an independent study (i.e., our relevant initial work can be found at [12]). For the same reason, the PoP Resource Allocation and PoP Network Controller components implement the functionalities of the DC Slice Controllers and WAN Slice Controllers, respectively.
We start by discussing the prototype with the functional description of the required steps for the slice deployment and then give an overview of the deployment workflow along with basic implementation details of the involved components.

The slice deployment operation involves the following steps:

- Slice Requirements Specification: The Tenant defines the slice requirements that include: (i) general slice parameters, including geographic constraints, cost model to use, monitoring options and slice time-frame (e.g., duration); (ii) a service graph consisting of service elements and links with particular demands for physical and/or virtual resources (e.g., VIM type and configuration); (iii) slice stitching requirements, such as bandwidth demands and resource reservation or tunneling protocol to use.

- Slice Embedding: In this slice deployment step, our facility involves the Marketplace to take initial decisions for the slice and dynamically discover physical resources that match the expressed demands. Practically, it defines the number of slice-segments and how to distribute the service elements in them, collects the resource offers from different Infrastructure and WAN Providers, and decides which of them to accept.

- Physical Resource Allocation: This step involves the allocation and booting up of physical servers and network devices in the different slice-segments. It follows the deployment of the required Operating Systems and VIMs. It completes with the booting up of all physical resources. We emulate the edge routers with diverse communication capabilities with physical machines supporting tunnelling protocols and bandwidth throttling.

- Slice Stitching: The facility stitches the slice-segments together by establishing the WAN connection between them and the required intra-domain network configurations. Furthermore, it employs the VLAN or VXLAN protocols for the slice isolation. At the end of this step, all slice servers are accessible between each other with private IP addresses.

- Service Deployment: This step involves the service deployment, which includes the transferring of the VM images to the particular servers, the creation of the VMs, and their booting up. The service activation usually completes with additional configuration processes for the service to operate.

- Monitoring Activation: The last step covers the deployment and configuration of the requested monitoring capabilities from the Tenant. It is related to the activation of the requested Key Performance Indicators (KPIs) from the Tenant, but also the deployment of a particular monitoring tool, e.g., Lattice [13] or Prometheus (https://prometheus.io).

We now present an overview of the slice deployment workflow (i.e., as shown in Fig. 2) and the basic implementation details of the prototype components. The Tenant realizes the Slice Requirements Specification through a GUI, which produces the Service Definition schema elaborated in [12]. 


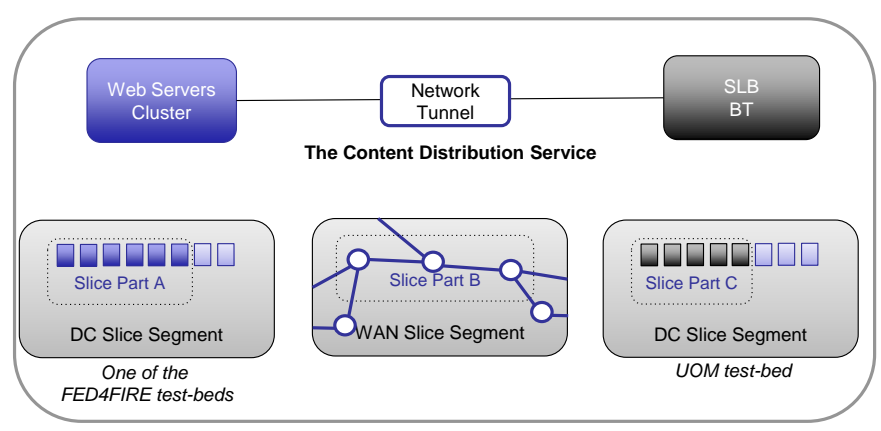

Fig. 3: Content distribution service slice

The Slice Specifications Processor, the Slice Builder and the PoP Resource Discovery components jointly implement the Slice Embedding step. The PoP Resource Allocation allocates on-demand the physical machines and deploys the requested VIM. The last two components act as wrappers of the novel FED4FIRE [14] and GENI [15] testbed tools (i.e., jfed CLI and geni-lib), in a similar approach to the work reported in [16]. Furthermore, the same components access a similar testbed control abstraction handling our own UOM test-bed. After the allocation of physical machines deployed at the different PoPs (i.e., slice-segments), the Slice Builder communicates with the Slice Resource Orchestrator (SRO), which in turn realizes the Service Deployment and Monitoring Activation through Ansible scripts. Lastly, the SRO oversees the Slice Operation; however, this is not part of the current work. The PoP Resource Allocation, PoP Resource Discovery and PoP Network Controller have been implemented in Python, while the other NECOS components as Node-RED nodes ${ }^{1}$. All of them exchange information descriptors specified as JSON messages, in accordance to the NECOS information model [12].

\section{ExPERIMENTAL EVALUATION}

\section{A. Evaluation Environment}

In this section, we validate experimentally the aforementioned slice deployment steps, namely the Slice Embedding (SEmb), Physical Resource Allocation (PRA), Slice Stitching (SS), Service Deployment (SDepl) and Monitoring Activation (MAct) processes, through our prototype implementation of the NECOS architecture. We consider a distributed content service example that spans geographically over Europe and USA. We assume that a Tenant requests a slice consisting of the following service functions: (i) a cluster of Web servers; (ii) a service load balancer (SLB); and (iii) benchmarking tools (BT). We further consider that the Tenant specifies each service function to be allocated in a particular geographic location, and thus, the service functions are mapped to two different DC slice segments, as depicted in Fig. 3. In particular, the left DC slice segment contains resources from the FED4FIRE testbeds federation, whilst the right DC

\footnotetext{
${ }^{1}$ https://nodered.org/
}

slice segment consists of physical resources located at our own UOM test-bed. To emulate the WAN slice segment that stitches the aforementioned DC slice segments, we deploy an additional physical machine at each side to serve as an edge router.

To proceed with the slice deployment, we defined the service and slice requirements as a generic YAML sliceinformation input that includes the three slice segments. In more detail, the DC slice segment at the UOM test-bed consists of six physical nodes hosting: (i) a service load balancer which distributes (i.e., in a round robin fashion) the Web traffic of a number of clients to the Web servers located at the left-side DC slice segment; and (ii) the benchmarking tools emulating the clients' behavior. The DC slice segment with the Web servers' cluster is physically located at the USA (i.e., the CloudLab Utah test-bed) and is accessed through the FED4FIRE facility. The number of physical machines in this segment constitutes the parameter Nodes of our experiment which ranges in $[5 \ldots 30]$. We choose two classes of Nodes' hardware type, i.e., the $p c 3000$ class with $3.0 \mathrm{GHz}$ processor, 2 GB DDR2 RAM and $300 \mathrm{~GB}$ storage, and the $d 430$ class with two $2.4 \mathrm{GHz} 8$-core processors, 64 GB DDR4 RAM and 2.2 TB storage (a detailed description of hardware specifications can be found at https://wiki.emulab.net/wiki/UtahHardware). The first class could serve as edge cloud and the latter as core cloud nodes. In this particular DC slice segment we consider the deployment of a Web server per physical node, we define the virtualization technology (i.e., Click-OS), and we specify service resource flavor (i.e., CPU, RAM utilization and storage usage) for each Web server. Furthermore, we designate the traffic disturbed policy (i.e., equally, randomly) that the service follows. The third slice segment (WAN), is responsible of configuring the inter/intra connectivity of the DC slice segments. We boot an extra physical machine in each DC slice side, that operates as an edge router, and we apply a GRE tunnel between them to resolve their connectivity. To secure intra connectivity we configure each physical node route table so that to be feasible to communicate with the edge router of the other side. For inter connectivity we assume a star topology where each physical node is connected to the edge router (central node) and through the edge router to the remote DC slice segment (physical remote nodes).

For the Monitoring Activation step of slice deployment, we regard the configuration of the CollectD open-source monitoring tool. For the allocated physical resources, we enable the following KPIs: i) CPU and RAM usage; and ii) incoming/outgoing traffic. The tool collects the KPIs metrics every $20 \mathrm{sec}$ (interval time). Our experimental results follow.

\section{B. Experimental Results}

Our results report the slice deployment time when either core or edge cloud nodes are employed at the DC slice segment accommodating the Web servers' cluster. Fig. 4 provides both a general view of the total time required for the slice instatiation, as well as detailed information for the time that each of the Slice Embedding, Physical Resource Allocation, 


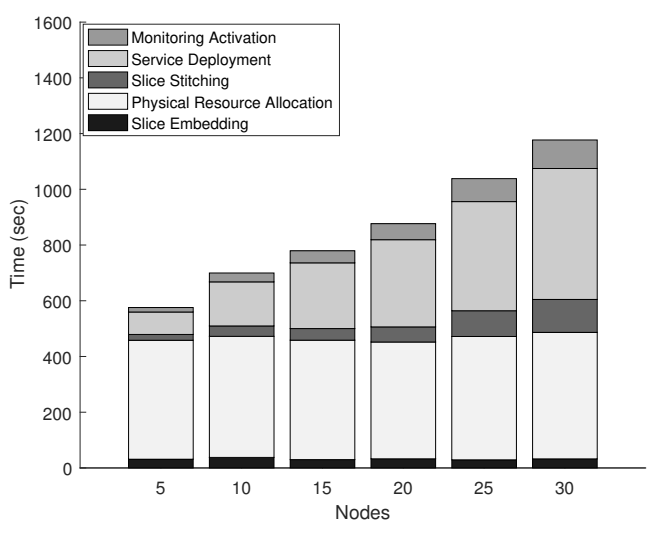

(a) Core cloud nodes

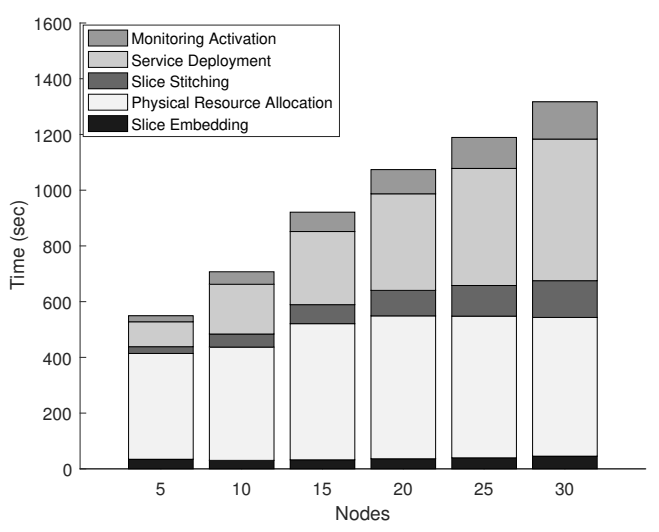

(b) Edge cloud nodes

Fig. 4: Slice deployment time

Slice Stitching, Service Deployment and Monitoring Activation steps requires. Time is expressed as a function of the number of the physical Nodes deployed at the CloudLab Utah test-bed. We repeat each experiment five times.

Fig. 4(a) and 4(b) indicate that the less time consuming steps are the Slice Embedding, the Slice Stitching and the Monitoring Activation. The first of them is found to be almost stable at around $30 \mathrm{sec}$ in case of core cloud nodes, and at around $35-40 \mathrm{sec}$ when edge cloud nodes are used. The exact values of time along with the standard deviations are further reported for clarity reasons in Table I. The Slice Stitching step ranges from almost $20-120 \mathrm{sec}$ and $25-130 \mathrm{sec}$, for core and edge cloud nodes, respectively. This step as well as the monitoring one linearly increase with the number of nodes. The Monitoring Activation starts at $16 \mathrm{sec}$ and goes up to almost $100 \mathrm{sec}$ in Fig. 4(a), whilst it ranges from 21 sec to $133 \mathrm{sec}$ in Fig. 4(b). These results show a slight time deterioration when edge cloud nodes are used in the DC slice segment. Especially the Slice Embedding step indicates almost none scalability limitation, at least of our experimental setup.

On the other hand, the Physical Resource Allocation and the Service Deployment steps are the most time consum-

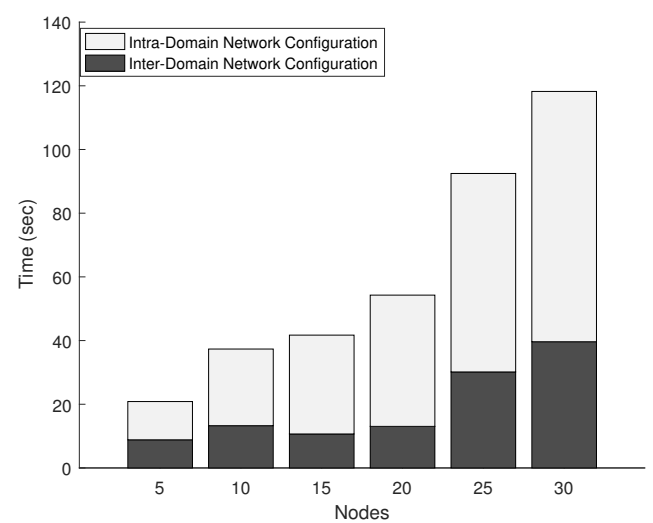

(a) Core cloud nodes

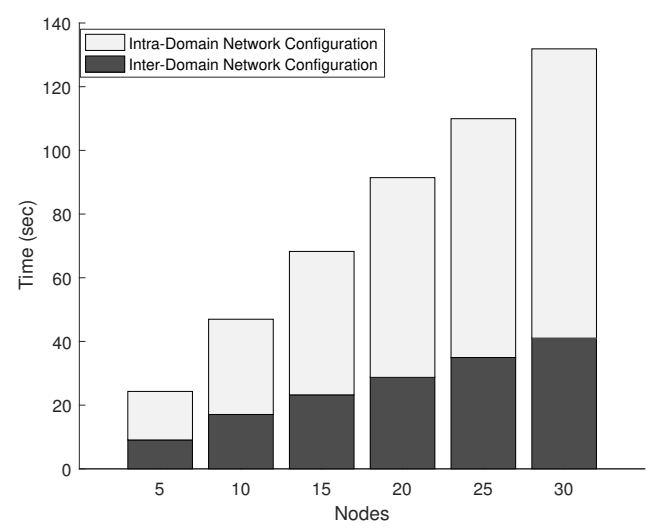

(b) Edge cloud nodes

Fig. 5: Slice stitching time

ing. Resource allocation involves the servers' boot-up time, which entails the prolongation of the general deployment time. However, in case of the core cloud nodes, the resource allocation requires as much as $74 \%$ of deployment time when Nodes $=5$, which significantly decreases at $30 \%$ when Nodes $=30$. The corresponding percentages range from $69-37 \%$ in case of edge cloud nodes. This decrease is due to the fact that the time remains almost stable with the increase of the nodes. Opposed to this observation, the requirements of the Service Deployment step in time increase with the number of nodes. As a result, service deployment time occupies $14-40 \%$ and $16-38 \%$ (in respect to the number of nodes) of the total slice deployment time when core and edge cloud nodes are employed, respectively.

In Fig. 5(a) and 5(b) we further elaborate on the Slice Stitching time requirements. As described in the experimental environment section, the WAN slice segment configures connectivity both inside the DC slice segments, and between the two geographically remote segments as well. In this figures, the light (dark) grey area indicates the intra(inter)domain network configuration. It is expected that the time requirements of the first would have been heavier compared 
TABLE I: Standard deviation of deployment time for core and edge cloud nodes

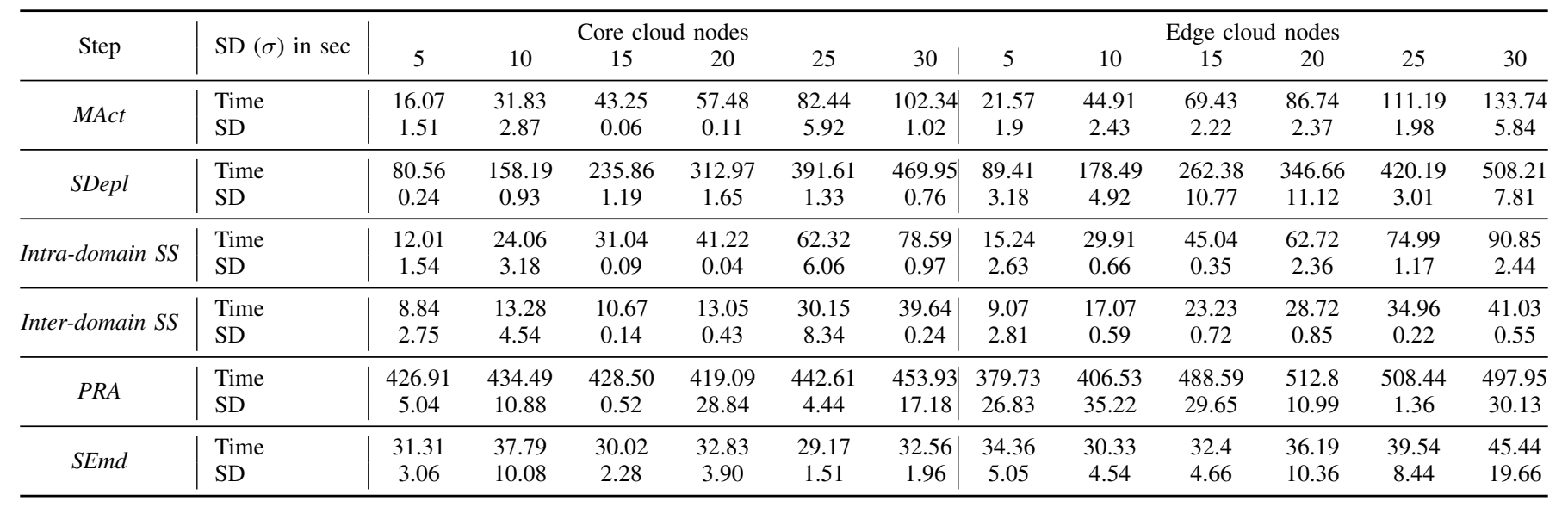

to the latter independently of the type of cloud nodes.

\section{CONClusions And Future WOrK}

In this paper, we studied the feasibility of multi-PoP slice deployment, motivated by the increasing interest in network slicing, as a means to provide inherent support for multiservice and multi-tenancy. We relied on a prototype implementation which provides support for slice and service deployment, based on the slice instantiation workflow exemplified in the NECOS architecture. Our results and micro-benchmarks across a diverse range of network slice sizes corroborate that slice instantiation delay scales linearly with the slice size. The dominant factor is physical resource allocation, which involves the booting of servers. In contrast to resource allocation (which is out of our control in the remote experimental infrastructures), both slice embedding and stitching incur low delays, and certainly do not introduce any scalability limitation, at least at the scale of our experimental setup.

In principle, there is room for optimizations (especially within the slice provider's domain) in order to reduce the slice instantiation delay. For example, slice instantiation tasks, executed across servers (e.g., virtual machine setup and configuration) and switches, can run in parallel to substantially speed up slice provisioning. We further plan to incorporate most sophisticated slice embedding mechanisms (e.g., [9]) and investigate the scalability of multi-domain slice instantiation with near-optimal slice embeddings.

\section{ACKNOWLEDGMENTS}

This work has received funding from the EU's Horizon 2020 research and innovation programme through the 4th open call scheme of the FED4FIRE+ (grant agr. n- 732638), the EU-BRA Horizon 2020 NECOS Project (grant agr. no 777067), and the MESON project, funded by the Management and Implementation Authority for Research, Technological Development and Innovation Actions (MIA-RTDI) in Greece (grant agr. no - T1EDK-02947).

\section{REFERENCES}

[1] X. Foukas, G. Patounas, A. Elmokashfi, and M. K. Marina, "Network slicing in 5G: Survey and challenges," IEEE Commun. Mag., vol. 55, no. 5, pp. 94-100, May 2017.
[2] F. S. D. Silva, M. O. O. Lemos, A. Medeiros, A. V. Neto et al., "NECOS project: Towards lightweight slicing of cloud federated infrastructures," in 4th IEEE Conf. on Network Softwarization and Workshops, June 2018, pp. 406-414.

[3] NFV White Paper, "Network Functions Virtualisation: An Introduction, Benefits, Enablers, Challenges \& Call for Action. Issue 1," Oct. 2012.

[4] M.-A. Kourtis, M. J. McGrath, G. Gardikis, G. Xilouris et al., "T-NOVA: An open-source MANO stack for NFV infrastructures," IEEE Trans. Netw. Service Manag., vol. 14, no. 3, pp. 586-602, Sept 2017.

[5] N. McKeown, T. Anderson, H. Balakrishnan, G. Parulkar et al., "Openflow: Enabling innovation in campus networks," SIGCOMM Comput. Commun. Rev., vol. 38, no. 2, pp. 69-74, Mar. 2008.

[6] N. Feamster, J. Rexford, and E. Zegura, "The road to SDN: An intellectual history of programmable networks," SIGCOMM Comput. Commun. Rev., vol. 44, no. 2, pp. 87-98, Apr. 2014.

[7] S. Clayman, "D3.1: NECOS System Architecture and Platform Specification.V1," 10 2018. [Online]. Available: http://www.h2020-necos.eu/documents/deliverables/ [Accessed: Jun. 19, 2019].

[8] L. A. Freitas, V. G. Braga, S. L. Corrła, L. Mamatas et al., "Slicing and allocation of transformable resources for the deployment of multiple virtualized infrastructure managers (VIMs)," in 4th IEEE Conf. on Network Softwarization and Workshops, June 2018, pp. 424-432.

[9] D. Dietrich, A. Rizk, and P. Papadimitriou, "Multi-provider virtual network embedding with limited information disclosure," IEEE Trans. Netw. Service Manag., vol. 12, no. 2, pp. 188-201, June 2015.

[10] G. Schaffrath, C. Werle, P. Papadimitriou, A. Feldmann et al., "Network virtualization architecture: Proposal and initial prototype," in Proc. of the 1st ACM Workshop on Virtualized Infrastructure Systems and Architectures, ser. VISA '09, 2009, pp. 63-72.

[11] P. Papadimitriou, I. Houidi, W. Louati, D. Zeghlache et al., "Towards large-scale network virtualization," in Wired/Wireless Internet Commun., 2012, pp. 13-25.

[12] P. D. M. Jr, F. L. Verdi, P. Valsamas, I. Sakellariou et al., "A marketplacebased approach to cloud network slice composition across multiple domains, submitted," in 4th IEEE Conf. on Network Softwarization and Workshops, June 2019.

[13] S. Clayman, A. Galis, and L. Mamatas, "Monitoring virtual networks with lattice," in 2010 IEEE/IFIP Network Operations and Management Symposium Workshops. IEEE, 2010, pp. 239-246.

[14] T. Wauters, B. Vermeulen, W. Vandenberghe, P. Demeester et al., "Federation of internet experimentation facilities: architecture and implementation," in European Conf. on Networks and Commun., Jun. 2014, pp. $1-5$.

[15] M. Berman, J. S. Chase, L. Landweber, A. Nakao et al., "Geni: A federated testbed for innovative network experiments," Computer Networks, Elsevier, vol. 61, pp. 5-23, Mar. 2014.

[16] P. Valsamas, I. Sakellariou, S. Petridou, and L. Mamatas, "A multidomain experimentation environment for $5 \mathrm{~g}$ media verticals," in IEEE INFOCOM Workshop on Computer and Networking Experimental Research using Testbeds 2019 (CNERT), April 2019. 